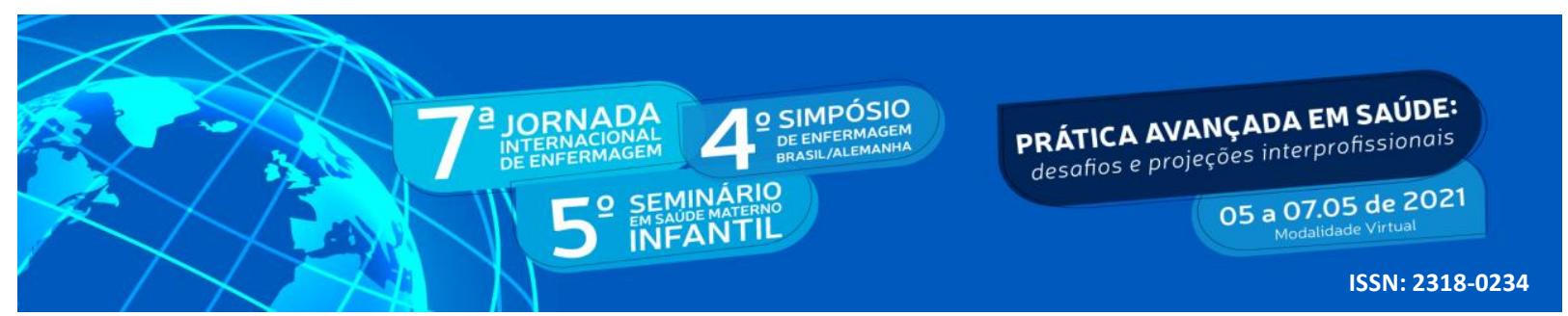

DOI: http://doi.org/10.48195/jie2021-012

\title{
IMPACTOS DA CONSTRUÇÃO DE POSTS INFORMATIVOS E EDUCATIVOS SOBRE SARCOPENIA DURANTE A PANDEMIA ${ }^{1}$
}

\author{
Júlia Schmitt Neves² ${ }^{2}$ Luciana Mello $^{3}$; Tereza Cristina Blasi ${ }^{4}$
}

\begin{abstract}
RESUMO
Objetivo: O trabalho visa relatar a experiência dos impactos da construção de posts informativos e educativos sobre Sarcopenia durante a pandemia. Método: Ocorreu de maio a junho de dois mil e vinte e teve como público-alvo a população idosa. Para a execução do material, as acadêmicas reuniram-se por meio de plataformas digitais. As pesquisas foram realizadas em livros, artigos científicos atualizados e na nova diretriz publicada pelo Grupo de Trabalho Europeu sobre Sarcopenia em Pessoas Idosas. O programa Canva ${ }^{\circledR}$ foi o meio para a construção dos 12 posts, com divulgação em redes sociais. Resultados: Obteve-se retorno e reações positivas em relação ao seu conteúdo. Conclusão: Conclui-se que o compartilhamento de informações científicas acessíveis são fundamentais para articulação entre ensino, serviço e comunidade visando as temáticas relacionadas a Nutrição do Idoso e a pandemia.
\end{abstract}

Palavras-chave: Educação Alimentar e Nutricional; Promoção da Saúde; Nutrição do Idoso.

\begin{abstract}
The work aims to report the experience of the impacts of the construction of informative and educational posts on Sarcopenia during the pandemic. It took place from May to June of two thousand and twenty and was aimed at the elderly population. For the execution of the material, the academics met through digital platforms. The research was carried out in books, updated scientific articles and in the new guideline published by the European Working Group on Sarcopenia in the Elderly. The Canva ${ }^{\circledR}$ program was the means for the construction of the 12 posts, with dissemination on social networks. Feedback and positive reactions were obtained regarding its content. It is concluded that the sharing of accessible scientific information is fundamental for the articulation between teaching, service and community, aiming at themes related to Elderly Nutrition and the pandemic.
\end{abstract}

Key Words: Food and Nutrition Education; Health Promotion; Elderly Nutrition.

\footnotetext{
1 Relato de experiência de acadêmicas do Curso de Nutrição da Universidade Franciscana - UFN.

2 Estudante do Curso de Nutrição e bolsista do PET - Saúde - Interprofissionalidade. Universidade Franciscana.

E-mail: juliaaschmitt@ outlook.com

3 Estudante do Curso de Nutrição. Universidade Franciscana. E-mail: mellu.sm@gmail.com

${ }^{4}$ Orientador. Professora do curso de Nutrição - UFN. Especialista em Terapia Nutricional - UFSC. Mestre em Ciências e Tecnologia de alimentos - UFSM. Coordenadora do grupo Assistência Multidisciplinar Integrada aos Cuidadores de Pessoas com Alzheimer - AMICA. E-mail: terezacristina@ufn.edu.br
} 


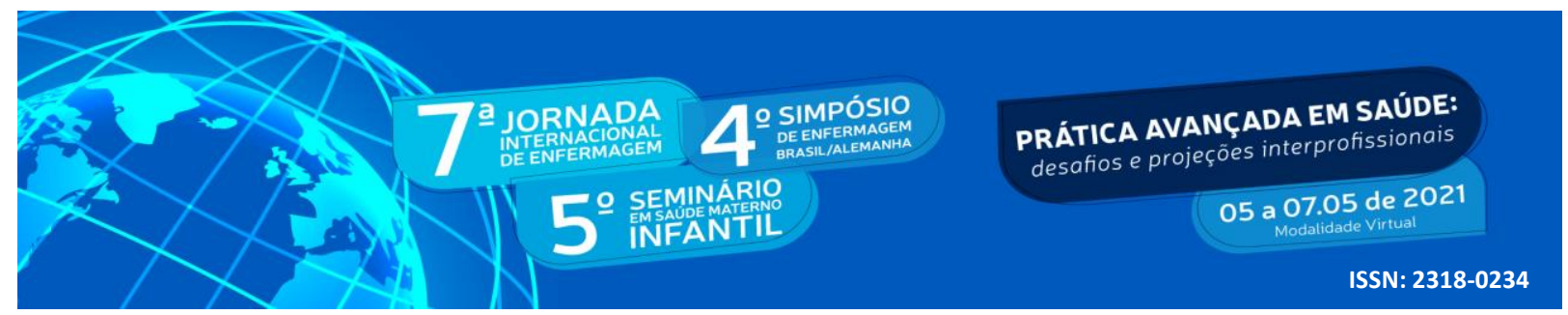

\section{INTRODUÇÃO}

O envelhecer é um processo que faz parte naturalmente da vida de qualquer indivíduo. Para isso acontecer de maneira saudável mostra-se como um grande desafio, já que o país está envelhecendo em ritmo acelerado e sem o planejamento adequado visando a qualidade de vida (PHILIPPI; AQUINO, 2017).

Uma das principais mudanças que ocorre com o avançar da idade é a sarcopenia. Essa se instala quando ocorrem, simultaneamente, perda de massa, força e qualidade musculares. Como consequência, pode levar ao aumento de quedas e fraturas, elevando o risco de imobilidade e de internações hospitalares. Além disso, a prevalência da doença em indivíduos entre 60 e 70 anos pode variar de 5 a 13\% e acima dos 80 anos, de 11 a 50\% (GARCIA et al., 2018; CRUZ-JENTOFT et al., 2019).

Além disso, não se pode deixar de relacionar as escolhas alimentares ao processo de envelhecimento. A partir disso, a Educação Alimentar e Nutricional (EAN) surge como uma ferramenta para divulgar conhecimento e informações com o intuito de impactar positivamente a saúde dos idosos. Assim, através dela é possível difundir os mecanismos associativos entre sarcopenia e alimentação, visto que uma ingestão nutricional adequada é uma importante estratégia para preservar a massa muscular e a força durante esse período da vida (BRASIL, 2012; MITHAL et al., 2013).

No ano de 2020 desencadeou-se a pandemia em virtude da COVID-19. A doença, causada pelo novo coronavírus SARS-Cov-2, aumentou os desafios para a parcela mais idosa da população brasileira, já que são mais suscetíveis a complicações. Em função disso, o Ministério da Saúde (MS) sugere como uma das medidas não-farmacológicas eficazes para a não propagação do vírus o Distanciamento Social Ampliado (DSA). Consequentemente, impactando de forma positiva no não adoecimento da população (BRASIL, 2020).

Por tudo isso, ações educativas voltadas à população idosa em tempos de pandemia são fundamentais para garantir o acesso à informações seguras e eficazes, consequentemente auxiliando nas escolhas alimentares saudáveis para estimular o envelhecimento ativo e fortalecer as ações de promoção de saúde e prevenção (BRASIL, 2006). 


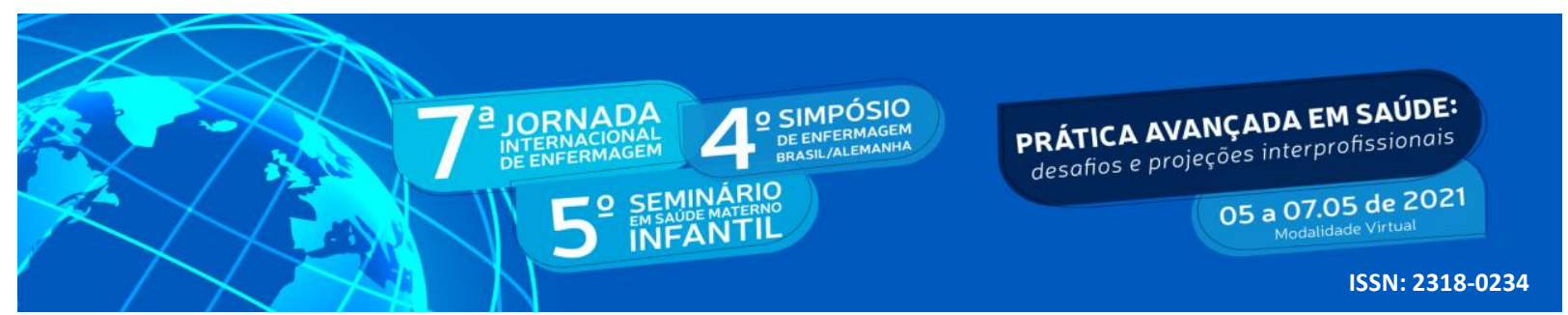

\section{OBJETIVO}

Relatar a experiência dos impactos da construção de posts informativos e educativos sobre sarcopenia durante a pandemia.

\section{METODOLOGIA}

O estudo trata-se de um relato de experiência das acadêmicas da Universidade Franciscana (UFN) a partir de um projeto extensionista da Disciplina de Nutrição do Adulto e do Idoso. Ocorreu no período de maio a junho de dois mil e vinte e teve como público-alvo a população idosa.

Para a execução do material, as acadêmicas reuniram-se por meio de plataformas digitais, aplicativo WhattsApp ${ }^{\circledR}$ na versão web e celular. O programa Microsoft Word $^{\circledR}$ foi utilizado para planejamento das postagens.

As pesquisas foram realizadas no livro Essências em Geriatria Clínica de Garcia et. al (2018), artigos científicos atualizados e na nova diretriz publicada pelo Grupo de Trabalho Europeu sobre Sarcopenia em Pessoas Idosas (EWGSOP, 2018).

Por fim, o programa Canva ${ }^{\circledR}$ foi o meio para a construção de posts educativos. A temática abordou a sarcopenia no processo de envelhecimento com enfoque na Nutrição do Idoso e teve como meio de divulgação as redes sociais do Curso de Nutrição da UFN.

\section{RESULTADOS E DISCUSSÃO}

No total desenvolveram-se 12 posts os quais contemplaram: O primeiro foi elaborado com a frase "Vamos falar de Sarcopenia?" para captar o público-alvo; O segundo abordou a definição da Sarcopenia; O terceiro, os três estágios da Sarcopenia os quais são: Provável Sarcopenia, Sarcopenia, Sarcopenia Grave; O quarto explicou sobre a classificação a qual é subdividida em primária ou secundária; O quinto informou os sinais para serem identificados: diminuição do equilíbrio, diminuição da velocidade de reação, diminuição da capacidade funcional e cognitiva, diminuição da massa e força musculares, diminuição da qualidade de 


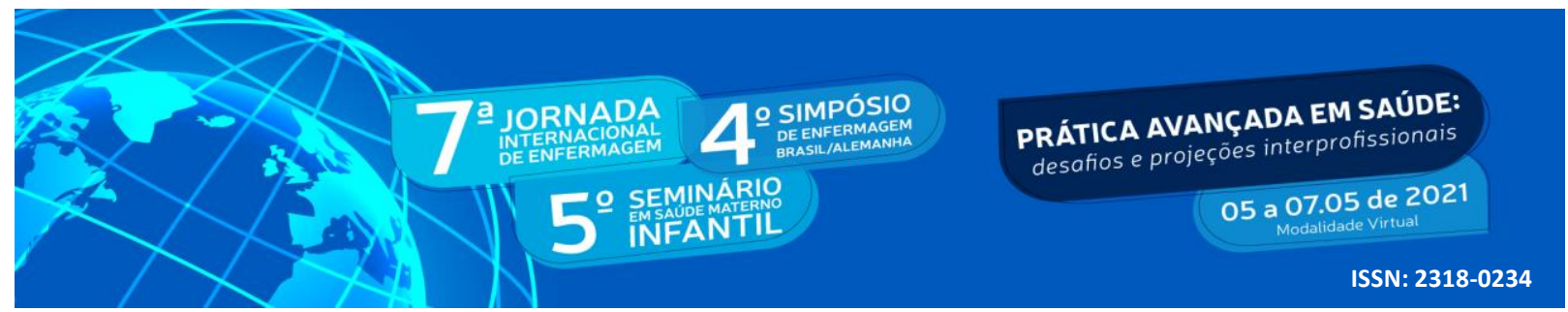

vida, aumento de quedas e fraturas, aumento da dependência ou necessidade de cuidados, aumento da sensação de fraqueza, aumento de dificuldades físicas e aumento da lentidão; $O$ sexto post instruiu sobre o papel dos nutrientes importantes na Sarcopenia: proteínas, vitamina D, ômega-3 e cálcio; O sétimo, o oitavo, o nono e o décimo exemplificaram as fontes alimentares em que são encontrados esses nutrientes; $\mathrm{O}$ décimo primeiro esclareceu que a prevenção ocorre por meio da associação entre atividade física e alimentação adequada; e, por fim, os créditos para elaboração do material educativo constituiu o décimo segundo post.

A construção de posts informativos sobre condições fisiológicas decorrentes do processo de envelhecimento, mostra que o desafio da EAN precisa ultrapassar os limites de ações dirigidas ao consumo, produção e abastecimento de alimentos. Dessa forma, estruturar, divulgar e integrar nas redes e mídia institucionais a informação apropriada, de modo a estimular a autonomia do sujeito para as práticas alimentares adequadas e saudáveis. Assim, possui o intuito de abranger iniciativas para a promoção da saúde (BRASIL, 2012).

A divulgação dos posts para a comunidade ocorreu através das redes sociais Facebook ${ }^{\circledR}$ e Instagram $^{\circledR}$ do Curso de Nutrição da UFN e por compartilhamento, nas mesmas redes sociais das autoras. Após a divulgação dos posts, houve retorno positivo em relação ao seu conteúdo, comprovada através dos seguintes relatos recebidos pelo WhattsApp ${ }^{\circledR}$ da professora: "Minha paciente está passando por esta fase, mas está muito bem e se alimentando normal..." e “Obrigado pelos ensinamentos... Continuamos aprendendo sempre." Além disso, reações positivas também foram deixadas através de comentários na página do Facebook $^{\circledR}$ do Curso de Nutrição da UFN.

Isso mostra que a adaptação durante a pandemia, situação diferente da habitual, é fundamental para manter a comunicação. Por esse motivo, a interação é essencial na área de saúde, pois por meio dela são obtidas informações valiosas para a condução de ações. Uma vez que, a diminuição da capacidade cognitiva ocorre no processo de envelhecimento e pode afetar a comunicação e a compreensão das pessoas idosas (BRASIL, 2006).

\section{CONCLUSÃO}

A construção de posts informativos e educativos sobre Sarcopenia durante a pandemia foi uma forma significativa de trazer alternativas para manutenção da saúde. O 


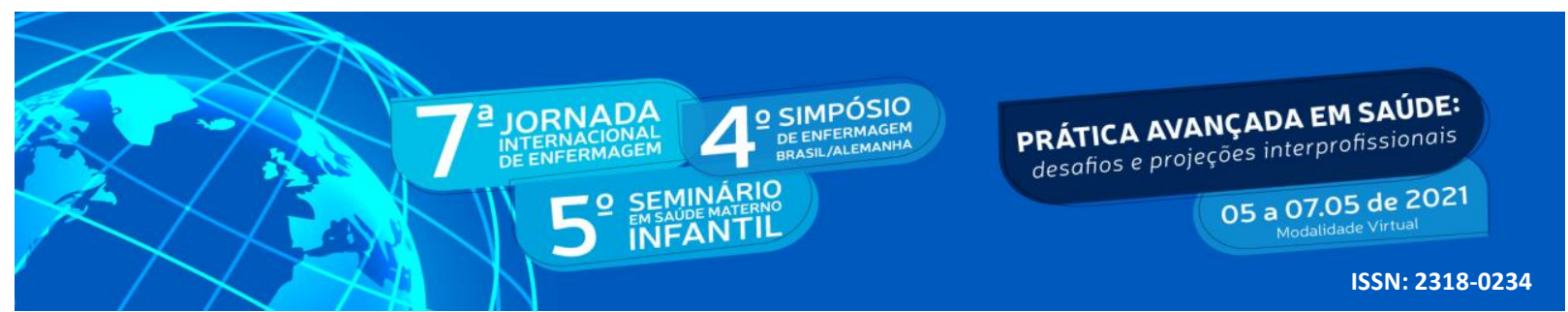

compartilhamento de informações científicas acessíveis foi importante para manter o idoso e seus cuidadores atentos a realizarem a alimentação de forma saudável e segura.

Além disso, mesmo em tempos de DSA, a busca contínua do conhecimento para a elaboração do material mostrou que as mudanças não impediram e nem prejudicaram a formação acadêmica. Consequentemente, contribuindo para a promoção de saúde da população.

A partir de tudo isso, projetos extensionistas são fundamentais para articulação entre ensino, serviço e comunidade visando as temáticas relacionadas a Nutrição do Idoso e a pandemia.

\section{REFERÊNCIAS}

BRASIL. Ministério do Desenvolvimento Social e Combate à Fome. Marco de referência de educação alimentar e nutricional para as políticas públicas. - Brasília, DF: MDS; Secretaria Nacional de Segurança Alimentar e Nutricional, 2012.

BRASIL. Ministério da Saúde. Secretaria de Atenção à Saúde. Departamento de Atenção Básica. Envelhecimento e saúde da pessoa idosa. Brasília: Ministério da Saúde, 2006.

BRASIL. Ministério da Saúde. Secretaria de Vigilância em Saúde. Centro de Operações Emergenciais em Saúde Pública. Doença pelo coronavírus 2019. Boletim Epidemiológico 7. 2020.

CRUZ-JENTOFT, Alfonso J et al. Sarcopenia: revised European consensus on definition and diagnosis. Age and ageing, v. 48, n. 1, p. 16-31, 2019.

GARCIA, E.; CIOCHETTA, D.I.; MENDES, D.S.; EVERLING, E.M.; SOUZA, S.P.; BEZERRA, O.S. Essências em Geriatria Clínica. Porto Alegre: EdiPUCRS, 2018.

MITHAL, A et al. Impact of nutrition on muscle mass, strength, and performance in older adults. Osteoporos Int., v. 24, n. 5, p. 1555-66, 2013.

PHILIPPI, Sonia Tucunduva; AQUINO, Rita de Cássia. Recomendações Nutricionais: nos estágios da vida e nas doenças não transmissíveis. Barueri, SP: Manole, 2017. 